\title{
Turbulent Structures and Coherence in the Atmospheric Surface Layer
}

\author{
K. Träumner • Th. Damian • \\ Ch. Stawiarski • A. Wieser
}

Received: 29 November 2013 / Accepted: 18 August 2014 / Published online: 4 September 2014

(C) The Author(s) 2014. This article is published with open access at Springerlink.com

\begin{abstract}
Organized structures in turbulent flow fields are a well-known and still fascinating phenomenon. Although these so-called coherent structures are obvious from visual inspection, quantitative assessment is a challenge and many aspects e.g., formation mechanisms and contribution to turbulent fluxes, are discussed controversially. During the "High Definition Clouds and Precipitation for Advancing Climate Prediction" Observational Prototype Experiment (HOPE) from April to May 2013, an advanced dual Doppler lidar technique was used to image the horizontal wind field near the surface for approximately $300 \mathrm{~h}$. A visual inspection method, as well as a two-dimensional integral length scale analysis, were performed to characterize the observations qualitatively and quantitatively. During situations with forcing due to shear, the wind fields showed characteristic patterns in the form of clearly bordered, elongated areas of enhanced or reduced wind speed, which can be associated with near-surface streaks. During calm situations with strong buoyancy forcing, open cell patterns in the horizontal divergence field were observed. The measurement technique used enables the calculation of integral length scales of both horizontal wind components in the streamwise and cross-stream directions. The individual length scales varied considerably during the observation period but were on average shorter during situations with $z / L<0$ compared to strongly stable situations. During unstable situations, which were dominated by wind fields with structures, the streamwise length scales increased with increasing wind speed, whereas the cross-stream length scales decreased. Consequently, the anisotropy increased from 1 for calm situations to values of $2-3$ for wind speeds of $8-10 \mathrm{~m} \mathrm{~s}^{-1}$. During neutral to stable situations, the eddies were on average quite isotropic in the horizontal plane.
\end{abstract}

Keywords Coherent structures · Dual Doppler lidar · Integral length scale

K. Träumner $(\varangle) \cdot$ Th. Damian · Ch. Stawiarski · A. Wieser

Karlsruhe Institute of Technology (KIT), Institute for Meteorology and Climate Research (IMK-TRO),

Hermann-von-Helmholtz-Platz 1, 76344 Eggenstein Leopoldshafen, Germany

e-mail: katja.traeumner@kit.edu 


\section{Introduction}

It is widely accepted that many, perhaps all, turbulent flows contain coherent motionsquasi-periodic patterns in the velocity components (e.g. Hussain 1983; Raupach et al. 1991; Takimoto et al. 2013). The scales of these patterns, also known as coherent structures, reach from the extent of the flow field itself down to the Kolmogorov scale, the smallest scale of turbulence. Hussain (1983) described such structures as "significant, but not necessarily the predominant, features" in the flow field. Often, they can be clearly identified by visual observation but their physical description is not obvious at all. Many questions are discussed controversially, such as their origin, possible formation mechanisms or contribution to transport processes. There exists an extensive literature in the field of fluid dynamics about this organization of turbulence, and reviews can be found, for example, in Robinson (1991) and Adrian (2007).

The atmospheric boundary layer, which ranges from the Earth's surface up to a height of several $100 \mathrm{~m}$ to few $\mathrm{km}$, can be described as a fluid with wall turbulence in a highReynolds-number regime. Therefore, it should contain different kinds of coherent structures. The most frequently observed are mixed-layer rolls that extend through the whole depth of the boundary layer with length scales of several km (e.g. Etling and Brown 1993; Drobinski et al. 1998) and streaky structures, also described as low-speed streaks or near-surface streaks. A review on these kinds of organization was given by Young et al. (2002). The streaky structures manifest as organized regions or bands of low momentum in the surface layer, which are elongated in the streamwise direction. They have a much smaller extent compared to mixed-layer rolls with only 100-200 m vertical extent and 500-2,000 $\mathrm{m}$ in the streamwise direction (e.g. Moeng and Sullivan 1994; Kim and Park 2003; Newsom et al. 2008; Iwai 2008). Drobinski and Foster (2003) referred the lifetimes of the streaks to several tens of min. Streaky structures are associated with shear and can be found in unstable as well as neutral to slightly stable stratification (e.g. Moeng and Sullivan 1994; Kim and Park 2003). The most descriptive approach to the evolution of such structures is the packet-structure model of Adrian (2007) who interprets the streaky structures as groups of hairpin vortices. Young et al. (2002) described two more theories: the first is a self-sustaining process in which streaks were generated by near-surface vortices and develop a dynamic instability. The second mechanism is based on optimal perturbation theory. The idea is that a properly configured initial perturbation can grow to counter-rotating vortices that are tilted into the mean shear. Kim and Park (2003) and Iwai (2008), for example, mentioned a relationship between low-speed streak formation and the development of large-scale horizontal rolls. Both, the mixed-layer rolls and the streaks, can be linked to microfronts, which can be observed in sweep-and-ejection patterns or ramp-like patterns in the time series of wind velocity, temperature, and humidity (e.g. Barthlott et al. 2007; Fresquet et al. 2009; Zhang et al. 2010; Takimoto et al. 2013; Zeeman et al. 2013).

Rarely described is a second kind of structure in the boundary layer: cells or alveolar structures in the vertical wind field. The original idea behind alveolar structures relates to Rayleigh-Bénard convection cells (Rayleigh 1916). Above the boundary layer, this phenomenon is well-known in cloud structures over the ocean in terms of closed-cell convection (updrafts and therefore clouds in the middle of the cells) and open-cell convection (updrafts at the boundaries). Thereby, closed cellular structure is preferred by cloud systems driven by cooling at the upper boundary, and open cell structure by systems driven by surface heating (Feingold et al. 2012). In the vertical wind field in large-eddy simulations (LES) without a background wind, these open cells are also visible in the lower boundary layer (e.g. Hellsten and Zilitinkevich 2013). The formation of the open cells would be in accordance with 
the surface heating from the ground. But up to now, the existence of those patterns in the atmospheric surface layer is not confirmed by measurements.

What can be observed depends strongly upon the tools used. Simulations are used most frequently (e.g. Moeng and Sullivan 1994; Kim and Park 2003; Drobinski and Foster 2003; Fresquet et al. 2009; Hellsten and Zilitinkevich 2013), and that allow most detailed insights because all relevant parameters can be displayed in all three dimensions for each timestep. However, simulations lack the most critical points-how real are the model results, how well can the underlying algorithms represent the real world? Laboratory studies e.g., in wind tunnels, with their short time and spatial scales allow three-dimensional reproductions of the velocity field e.g., by using particle image velocimetry techniques (Takimoto et al. 2013) or laser Doppler anemometry (Ruck 1987), but the dimensions are typically not sufficient to reproduce atmospheric conditions. Both tools allow a comprehensive characterization of the structures and their interaction with the environment. However, despite the advantages of numerical and physical models, they need a reliable verification with atmospheric measurements.

For coherent structure investigation in the atmospheric boundary layer, the standard set-up is the deployment of in situ instruments on towers (e.g. Barthlott et al. 2007; Zhang et al. 2010; Segalini and Alfredsson 2012; Zeeman et al. 2013). The sweep-and-ejection viewpoint of coherent structures in meteorology is determined by this technique. The approach enables statistically proven conclusions about the one-dimensional behaviour (length, separation, intensity) during different atmospheric conditions, and an estimate of the contribution of the coherent structures to turbulent fluxes. However, it is difficult to deduce from the ramp-like patterns the shape and two- or even three-dimensional extent of the structures. Also, it is not possible to track individual structures and observe their evolution.

Different attempts at overcoming this one-dimensionality have been made. Inagaki and Kanda (2010) used a set of 40 sonic anemometers to measure wind-speed fluctuations over a test field site with very large roughness. Using Taylor's hypothesis of frozen turbulence, they were able to generate a two-dimensional picture of velocity fluctuations. In addition to in situ instruments, remote sensing instruments have been used. First attempts at using Doppler lidar to study coherent structures were made by Drobinski et al. (1998) who examined boundary-layer rolls and Drobinski et al. (2004) who showed streaks in the radial velocity field of a Doppler lidar. To gain a more complete picture of the individual wind components, dual Doppler lidar techniques are an option. This attempt was first realized by Newsom et al. (2008) during the Joint Urban 2003 field experiment. They used two lidars to span a triangular area of approximately $3 \mathrm{~km}^{2}$ and a scan technique that allowed horizontal windfield reproduction with a temporal resolution of $30 \mathrm{~s}$. They demonstrated the qualitative behaviour of the streamwise and the cross-stream wind components during two situations with unstable, one with neutral and one with weakly stable conditions and estimated the corresponding length scales. Dual Doppler lidar was also used by Iwai (2008) to demonstrate the interaction between convective rolls and near-surface streaks. Both studies demonstrated that dual Doppler lidar is highly promising for coherent structure research, particularly for streaky structures. However, this measurement approach is complex and statistically proven implications up to now are not available.

In order to complement this short overview it should be mentioned, that airborne measurements have also been made to capture coherent structures in higher regions of the boundary layer, for example by Etling and Brown (1993), Hartmann et al. (1997), Hasel et al. (2005).

At the Institute for Meteorology and Climate Research at the Karlsruhe Institute of Technology (KIT) the mobile integrated observation system KITcube has been in operation since 2011 (Kalthoff et al. 2013). Amongst other state-of-the-art meteorological instruments 
KITcube involves two long-range Doppler lidars. Using an optimized dual Doppler approach, this technique was for the first time systematically conducted over a time period of several weeks. The results give impressive insights into the behaviour of the near-surface flow. In this paper, we give a qualitative description of the different kinds of patterns that can occur and a quantitative characterization of the coherence using integral length scales. The measurement set-up and the data handling procedure are described in Sect. 2. Section 3 gives impressions of the discovered patterns and a qualitative analysis of the occurring periodic and aperiodic structures. A two-dimensional autocorrelation algorithm was used to receive quantitative characteristics of the coherence as integral length scales and anisotropy coefficients. Their dependence on stability as well as the mean wind is given in Sect. 4. In Sect. 5, conclusions are drawn.

\section{Experimental Set-up and Data Evaluation}

\subsection{Field Study}

Measurements used herein were embedded in the field campaign HOPE (HD $(C P)^{2}$ Observational Prototype Experiment), which was part of the project "High Definition Clouds and Precipitation for Advancing Climate Prediction". HOPE took place near Jülich, Germany, from April to May 2013, and Fig. 1 gives an overview of the measurement site. The local area has a rural character, where villages alternate with farmland. There are two large open cast mining areas to the south-west and the north-east of the measurement site, both several hundreds of $\mathrm{m}$ deep, the one in the north-east with a dump of more than $200 \mathrm{~m}$ height. The dump is covered with trees. Within the measurement area, several rows of trees separate the individual agricultural fields.

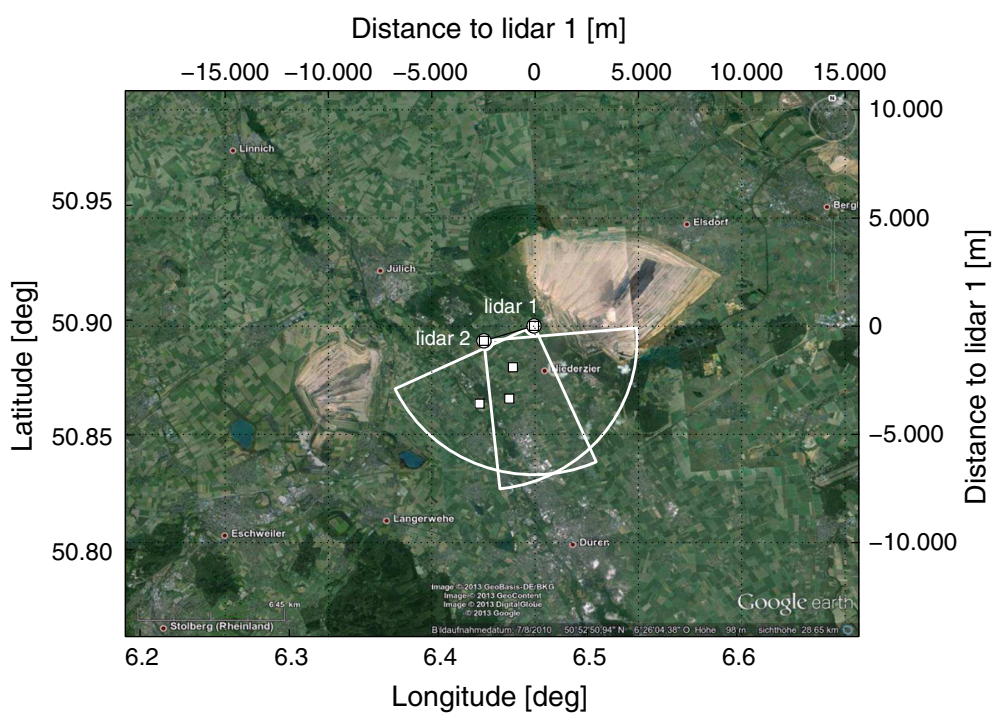

Fig. 1 Overview of the landscape at the HOPE site and the scan pattern: the dots mark the lidar locations, the squares mark the locations of the energy balance stations (aerial photography with permission from geocontent $\mathrm{GmbH})$ 
Table 1 Technical

characteristics of the two

"WindTracer" lidar systems

\begin{tabular}{lll}
\hline "WindTracer" & 1 & 2 \\
\hline Year of construction & 2009 & 2004 \\
Type of laser & Er:YAG & Tm:LuAG \\
Wavelength (nm) & 1,617 & 2,023 \\
Pulse length (ns) & 300 & 370 \\
Pulse energy (mJ) & 2.7 & 2.0 \\
Pulse repetition frequency (Hz) & 750 & 500 \\
Sampling rate (MHz) & 250 & 250 \\
\hline
\end{tabular}

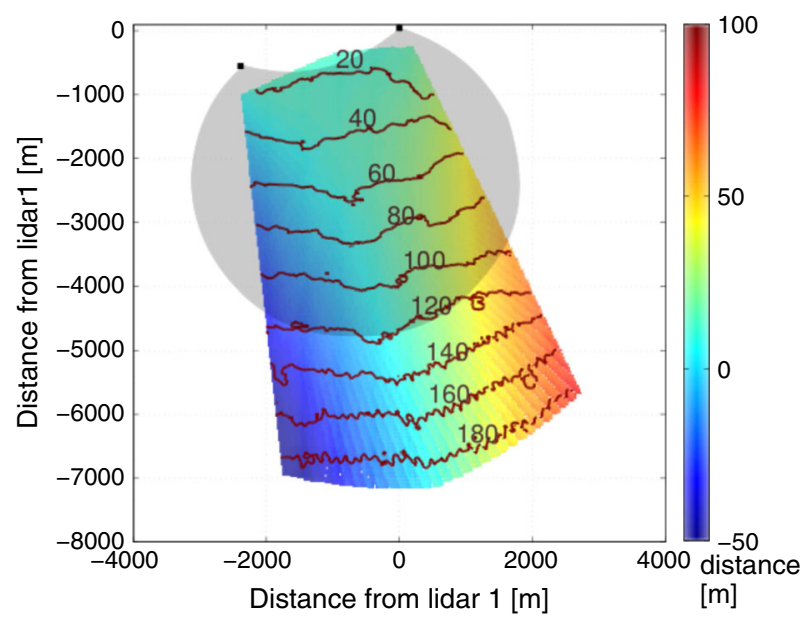

Fig. 2 Distance (colours) between the two lidar planes and height above ground of the mean measurement height (contour lines). The shaded area is considered for further evaluation

For wind-field measurements, two Doppler lidars "WindTracer" (manufactured by Lockheed Martin Coherent Technologies Inc., Louisville, Colorado, USA) were separated approximately $2.5 \mathrm{~km}$ apart and operated as a dual Doppler system. Table 1 summarizes parameters of the lidar systems. The range gate lengths as well as the range gate centres can be freely arranged starting at about $400 \mathrm{~m}$ distance from the instrument. With a mid-European aerosol load of the atmosphere, the instruments are able to measure up to distances of $12-15 \mathrm{~km}$ within the boundary layer. Both instruments have a two-axis scanner, which allows freely defined patterns throughout the whole upper hemisphere. Scanner movements can be performed with different speeds for both axes at the same time. A specially designed control system for the two lidar systems (Stawiarski et al. 2013) allows highly synchronized scan patterns as well as real time adaptation of scan parameters to atmospheric conditions e.g., the current wind speed.

Both lidar systems performed a synchronized low-elevation plan-position indicator (PPI) scan pattern. The azimuth ranged from $155^{\circ}$ to $245^{\circ}$ for the system indicated as 'lidar $1^{\text {' and }}$ $84^{\circ}$ to $174^{\circ}$ for the system indicated as 'lidar 2', respectively (Fig. 1). Due to obstacles in the line-of-sight (houses in a nearby village, individual trees, a power line) it was not possible to scan at zero elevation. To avoid largely varying distances between the two lidar-scanning planes and to enable lower measurement altitudes, the elevation angle was adapted as a function of the azimuth angle (a constant elevation angle would result in higher measurement heights). This method yields slightly curved planes. Figure 2 shows the distance between both 
lidar planes as well as the mean distance to the surface. In spite of the elevation used, some hard targets remained and had to be taken into account (see Sect. 2.2).

The scan velocity of both instruments was adapted to the mean wind speed on an hourly basis to fulfill the condition

$$
\begin{aligned}
& \text { If } \sqrt{\bar{u} C_{s}} \geq \Delta r_{\min }: t_{0_{\mathrm{opt}}}=\sqrt{C_{s} / \bar{u}} \\
& \text { If } \sqrt{\bar{u} C_{s}}<\Delta r_{\min }: \quad t_{0_{\mathrm{opt}}}=C_{s} / \Delta r_{\min }
\end{aligned}
$$

where $\bar{u}$ is the mean wind speed, $t_{0}$ is the time interval needed to sweep through the area (for both lidars), $\Delta r_{\text {min }}$ is the minimal range gate length, which depends on the lidar pulse width, $C_{s}=(2 \pi / 360) \beta d / f$ where $d$ is the maximal evaluated distance, $\beta$ is the full planar azimuth angle, and $f$ is the measurement frequency. This secured a minimization of the time sampling error (Stawiarski et al. 2013). Wind-speed profiles were obtained from a full PPI scan that was performed by both lidars at the beginning of each hour using a velocity-azimuth display (VAD) algorithm (Browning and Wexler 1968). For values of $\bar{u}$, the mean wind speed between the surface and $200 \mathrm{~m}$ above the ground was used.

Complementary data from KITcube were gained from "Windcube" Doppler lidars (manufactured by Leosphere), in situ measurements from a 30-m tower as well as from two energy balance stations (for details about the instruments see Kalthoff et al. 2013). Three more energy balance stations, which were operated by the Institut Agrosphere, Forschungszentrum Jülich and the Institut für Geophysik und Meteorologie, University of Cologne, complement the measurements. The 30-m tower and one of the "Windcube" lidars were located near 'lidar 1', two additional "Windcubes" were located near 'lidar 2'. The energy balance stations were distributed over the field site (Fig. 1).

\subsection{Data Handling}

The horizontal wind field was evaluated on a Cartesian grid, while the temporal resolution is the time for one synchronized sweep though the area, which was here approximately 12 $\mathrm{s}$. The spatial resolution of the grid was adapted to the temporal resolution by using the maximum of the distances between range gate centres of lidar 1 and 2, and the distances between two adjacent points $(2 \pi d / 360 f) \partial a z / \partial t$ at $d=4,000 \mathrm{~m}$ in beam direction. This means that the retrieval resolution was optimized to retrieve the wind field without any gaps (due to the algorithm) up to 4,000 m away from the instruments. The spatial resolution varied from 51 to $79 \mathrm{~m}$, depending on the scan speed and the range-gate setting used.

The wind vector $\mathbf{u}_{H}$ at each grid point $\mathbf{r}_{0}$ in the lidar overlap plane was obtained by minimizing the cost function

$$
J=\sum_{n} g_{n}\left(r v_{n}-\mathbf{u}_{H}\left(\mathbf{r}_{0}\right) \cdot \hat{\mathbf{r}}_{n}\right)^{2},
$$

where $r v_{n}$ are the radial wind components measured by both lidars with unit beam vectors $\hat{\mathbf{r}}_{n}$ (azimuth and elevation were considered) whose range-gate centres fall into an $R$ circle around $\mathbf{r}_{0}$ during the time interval of one complete sweep. $R$ is taken as the diagonal of the grid, and $g_{n}$ are additional relative weights of the individual velocity estimates that were chosen as the line segment length of the lidar beam that falls inside the cell. $n$ is the total number of measurements that fulfill the conditions (not the total number of lidars). Radial velocity measurements with a signal-to-noise ratio $<-10 \mathrm{~dB}$ were not considered. Additionally, a jump filter of $5 \mathrm{~m} \mathrm{~s}^{-1}$ was used to skip unreasonable radial wind velocities, which were not assigned by the signal-to-noise filter. $\mathbf{u}_{H}$ is here interpreted as the horizontal wind vector, 
which assumes that the vertical wind component can be neglected, and which in turn is justifiable for the elevations of less than $2.03^{\circ}$ used.

The propagation of the individual lidar errors due to uncorrelated noise to the retrieved wind components $\varepsilon_{u_{i}}^{2}$ has an upper limit of

$$
\varepsilon_{u_{i}}^{2} \leq \frac{1}{\sin ^{2}(\Delta \chi)} \epsilon_{r v}^{2}
$$

where $\Delta \chi$ is the angle measured from $\hat{\mathbf{r}}_{1}$ to $\hat{\mathbf{r}}_{2}$ and $\epsilon_{r v}^{2}$ is the uncorrelated noise for both lidars (Stawiarski et al. 2013). To avoid propagation errors that are too large, an upper limit of $\sin ^{-2}(\Delta \chi)=4$ was chosen, which implies that only intersection angles between $30^{\circ}$ and $150^{\circ}$ were considered. Figure 2 indicates the corresponding area with the shaded area.

Although the used scan pattern attempted to reduce the distance between the two lidar planes, there are still significant distances (between zero to about $40 \mathrm{~m}$ ) in most of the analysis domain (Fig. 2). To check if the distance has an effect on the retrieved wind, 15-min averaged wind speeds at $75 \mathrm{~m}$ above the ground were calculated for points in the overlap plane with a plane distance $<5 \mathrm{~m}$ on the one hand, and points with a plane distance $>40 \mathrm{~m}$ on the other hand. The results show that the differences between both wind speeds follow a Gaussian distribution with an expectation value of $(0.14 \pm 0.03) \mathrm{m} \mathrm{s}^{-1}$. This indicates slightly higher wind speeds for the points with the close-by planes, which corresponds to the expectation underlying a logarithmic wind profile. At once, it has to be considered that the points corresponding to the two conditions were located at different locations in the field i.e., also an orographic effect might be an explanation. Overall we rate the effect as negligible.

For each timestep, the mean horizontal wind speed and wind direction in the overlap area were calculated. A comparison of 15-min averaged values between retrieval data from the dual Doppler lidar measurements, and wind speed obtained by the "Windcube" Doppler lidars, as well as in situ tower measurements, was performed to check the retrieval results. The tower measurements represent the conditions at a height of $32 \mathrm{~m}$ a.g.1., the "Windcube" wind speed and direction were obtained using a VAD algorithm and are representative for $z=75 \mathrm{~m}$ a.g.l. The dual Doppler lidar retrieval value is a spatial and temporal average of measurements at heights between 10 and $120 \mathrm{~m}$ a.g.l. over an area of approximately $12 \mathrm{~km}^{2}$. The correlation coefficients between the retrieval results and the values obtained from the different instruments vary between 0.79 and 0.94 for the wind speed and 0.88 and 0.95 for the wind direction, respectively. Figure 3, left, shows the comparison of the instruments located at the site marked with 'lidar 2' in Fig. 1 and the field-averaged dual Doppler lidar retrieval values. Wind speeds $<6 \mathrm{~m} \mathrm{~s}^{-1}$ are slightly underestimated compared to the wind speed from the "Windcubes" and slightly overestimated compared to the in situ measurements at 32 $m$ a.g.l. (not shown here). For higher wind speeds, the retrieval underestimates the wind speed systematically at both locations although at location 'lidar 2' this is less pronounced. Figure 3, right, shows two examples of complementary wind profiles. It seems that the underestimation results from two effects: an orographic effect due to the comparison of a local measurement to a spatial mean, as well as a height-averaging effect due to the tilted lidar plane. The latter is considered in the data handling (see below). The orographic effect might arise from the complex terrain with several hundreds of $\mathrm{m}$ of height change due to the open cast mining areas. Especially at location 'lidar 1' some local circulations might arise.

To enable comparability between different situations, the wind vector was decomposed into the streamwise wind component $\left(u_{1}\right)$ and the cross-stream wind component $\left(u_{2}\right)$ using 

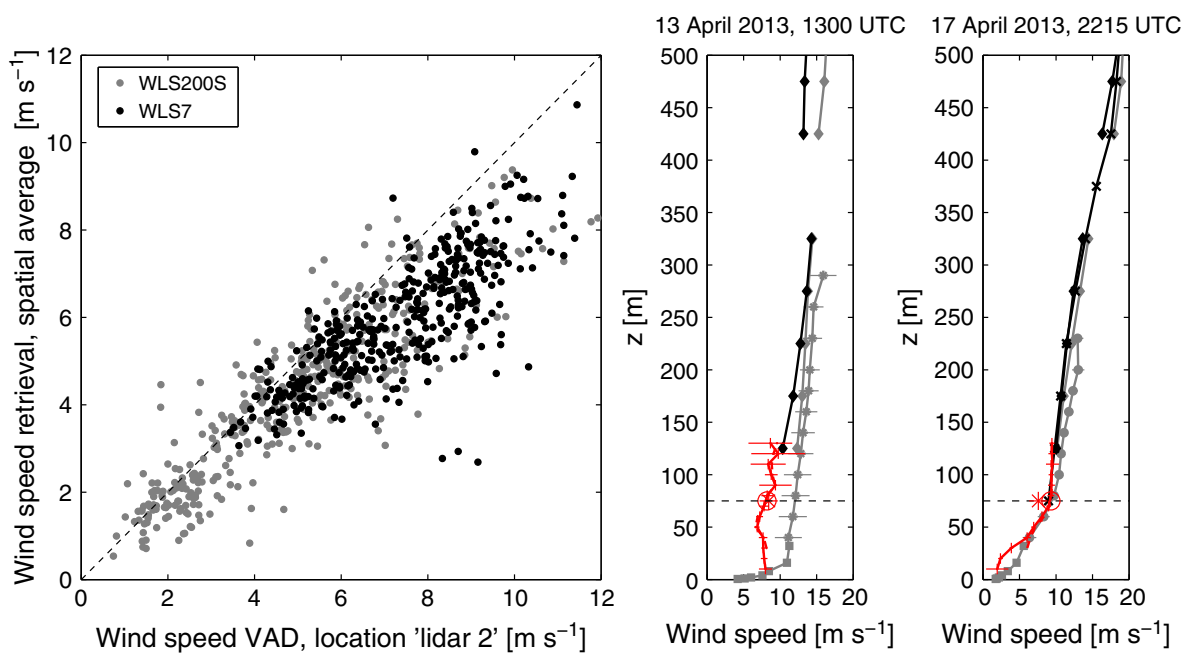

Fig. 3 Comparison between the mean wind speed of the retrieved wind field and the mean wind speed obtained by a WLS200S and a WLS7 "Windcube" Doppler lidar at site marked with 'lidar 2' (left) and two complementary profiles from both sites (right; gray symbols for site 'lidar 1': tower (square), WLS7 (small circle) and lidar 1 (diamond); black symbols for site 'lidar 2': WLS200S ( $\times$ ) and lidar 2 (diamond); and red symbols for the dual Doppler data: all values (line), only values with less than $5 \mathrm{~m}$ distance between the lidar planes (dashed line, hidden by the continuous line), 75-m averaged value (big open circle) and field-averaged value (asterisk). The horizontal lines mark the standard deviation intervals, the dashed lines indicate $75 \mathrm{~m}$ a.g.1.)

the spatial and temporal (15-min) averaged wind direction from the dual Doppler retrieval. Afterwards the fluctuations were calculated using the spatial and temporal means $u_{1}^{\prime}=$ $u_{1}-\overline{u_{1}}$ and $u_{2}^{\prime}=u_{2}-\overline{u_{2}}$, respectively. The remaining hard targets generate strong deviations, which were captured by a threshold filtering of values $\left|u_{i}^{\prime}\right|>5 \mathrm{~m} \mathrm{~s}^{-1}$.

Due to the tilted plane, which implies that height above the ground increases with distance from the instruments, a correction of $u_{i}^{\prime}$ was necessary. Figure $5 \mathrm{~b}$ gives an example of a stratified wind field without height correction. Using the mean measurement height (Fig. 2), 15-min averaged wind profiles for $u_{i}^{\prime}$ from the dual Doppler data were calculated (see also Fig. 3, right) and a third-order polynomial was fitted to the profiles. It can be observed that for the streamwise wind component the slopes range from $-0.1 \mathrm{~m} \mathrm{~s}^{-1}$ per $100 \mathrm{~m}$ (5th percentile) through $2.1 \mathrm{~m} \mathrm{~s}^{-1}$ per $100 \mathrm{~m}$ (50th percentile), and up to $5.0 \mathrm{~m} \mathrm{~s}^{-1}$ per $100 \mathrm{~m}$ (95th percentile). So, there are cases with a considerable effect due to the tilted plane. This trend is not observed for the cross-stream wind component, where the effect is on average negligible (50th percentile is $-0.6 \mathrm{~m} \mathrm{~s}^{-1}$ per $100 \mathrm{~m}$ ). Using the fitted profile, the wind components $u_{i}^{\prime}$ were corrected for the height effect according to their position in the lidar overlap plane. Without height correction, the estimated integral length scales elongate significantly with increasing slopes.

To deal with data gaps, an interpolation algorithm was applied. The algorithm used a linear interpolation of the eight surrounding neighbours of the grid point. The algorithm was applied to the wind field up to $5,000 \mathrm{~m}$ distance to the single instruments. Afterwards, the grid was rotated with wind direction i.e., the streamwise direction points along the $x$-axis. For this rotation, a bilinear interpolation algorithm was used i.e., the wind field is slightly smoothed. 
To quantify the coherence, a two-dimensional autocorrelation function was used,

$$
r_{u_{i}}(\tau, \eta)=\frac{1}{\sigma_{u_{i}}^{2}} \frac{1}{\left(N_{1}-|\tau|\right)\left(N_{2}-|\eta|\right)} \sum_{x=|\min (0, \tau)|}^{\min \left(N_{1}, N_{1}-\tau\right)} \sum_{y=|\min (0, \eta)|}^{\min \left(N_{2}, N_{2}-\eta\right)} u_{i}^{\prime}(x+\tau, y+\eta, t) u_{i}^{\prime}(x, y, t)
$$

with lags $\tau$ in the $x$-direction (streamwise) and $\eta$ in the $y$-direction (cross-stream) and grid points $(x, y)\left(N_{1,2}\right.$ are the dimensions of the grid). The variance $\sigma_{u_{i}}^{2}$ was calculated for the whole wind field, viz

$$
\sigma_{u_{i}}^{2}=\frac{1}{N_{1} N_{2}} \sum_{x} \sum_{y} u_{i}^{\prime}(x, y) .
$$

It should be mentioned here that from a strictly mathematically point of view the variance should be calculated from the same data as used in Eq. 4 to secure that the autocorrelation function is limited to the interval [ -11$]$.

Integral length scales are defined for a single direction e.g., for lags $\tau$, according to

$$
l=\int_{0}^{\infty} d \tau r_{u_{i}}(\tau)
$$

Of interest are the integral length scales in the streamwise and cross-stream directions. To evaluate this in the streamwise direction, the approach of Lenschow and Stankov (1986)

$$
l_{u_{i}, x}=\sum_{\tau=0}^{\tau_{0}} r_{u_{i}}(\tau, 0) \Delta \tau
$$

was used where $\tau_{0}$ is the first zero crossing point of the corresponding autocorrelation function $r_{u_{i}}$. Analogously for $l_{u_{i}, y}$,

$$
l_{u_{i}, y}=\sum_{\eta=0}^{\eta_{0}} r_{u_{i}}(0, \eta) \Delta \eta
$$

The ratio $l_{u_{i}, x} / l_{u_{i}, y}$ is a measure of isotropy, which should be unity for isotropic turbulence (Newsom et al. 2008).

To capture periodicity for lags between 0 and 2,000 m, each autocorrelation was analyzed for local maxima that exceed 0.2. To characterize the distance between structures, the distance from lag zero to the lag of the first local maximum was considered. Figure 4 shows an example to illustrate the procedure.

It is generally known, that coherent structures are affected by shear and stratification. Therefore a relationship between integral length scales and the parameter

$$
\xi=\frac{z}{L}=\frac{-k z g\left(\overline{w^{\prime} \theta^{\prime}}\right)_{s}}{\overline{\theta_{V}} u_{*}^{3}}
$$

was considered by e.g. Newsom et al. (2008) and Salesky et al. (2013). Here $L$ is the Obukhov length, $k$ is the von Karman constant, $g$ is the acceleration due to gravity, $\left(\overline{w^{\prime} \theta^{\prime}}\right)_{s}$ is the vertical kinematic potential temperature flux at the surface, $\overline{\theta_{V}}$ is the mean virtual potential temperature and $u_{*}$ is the friction velocity. $L$ was determined from data obtained from the energy balance stations using TK3 software (Mauder et al. 2013). The mounting of the instruments was $4 \mathrm{~m}$ above the ground, therefore $z=4 \mathrm{~m}$ was used. We refrained using 

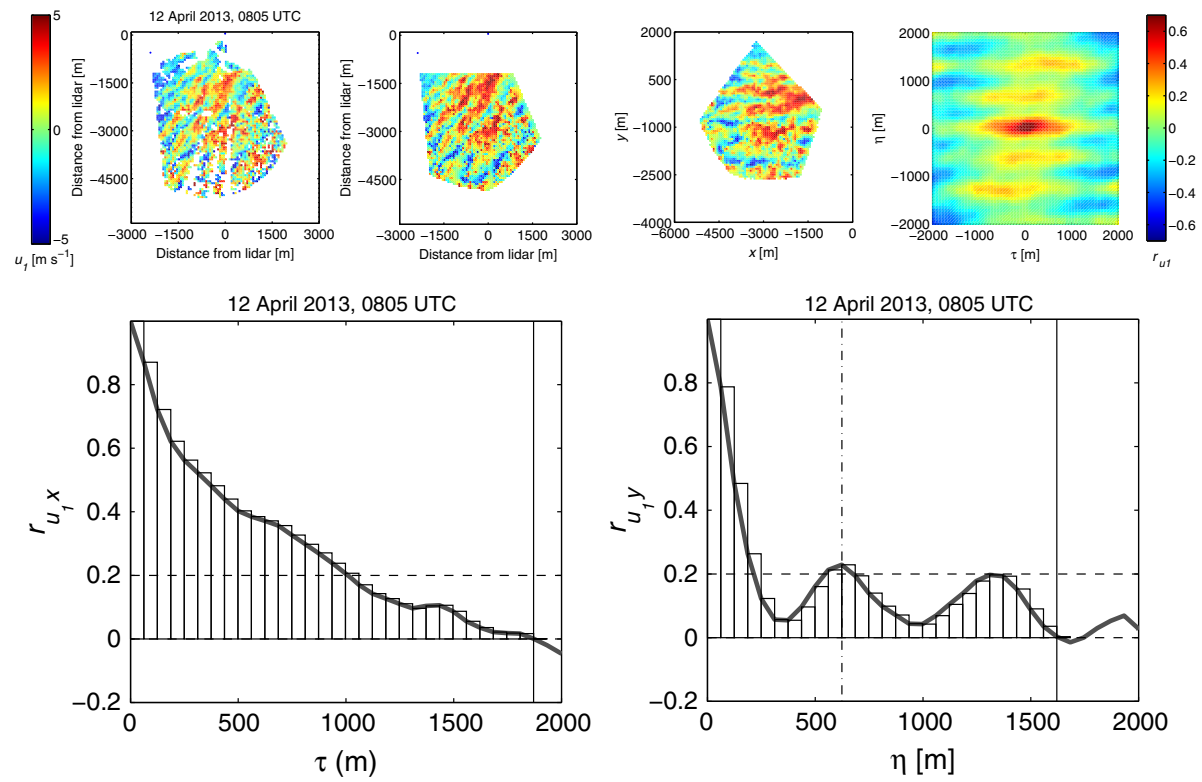

Fig. 4 Procedure to evaluate the coherence: wind field (top, first from left), height-corrected and interpolated (to fill the gaps) wind field (top, second from left), rotated wind field (top, third from left), and field of the two-dimensional autocorrelation function (top, right). At the bottom, the autocorrelation functions of the streamwise wind component evaluated in the streamwise direction (left) and in the cross-stream direction (right) are shown (the vertical line indicates the first zero crossing, the dashed vertical line the first additional maximum)

$z=z_{i}$, where $z_{i}$ is the boundary-layer height, because a reliable high resolution information, especially during night, was not available for the considered period. The five stations provide information over different plant cover, representing the typical plants in this rural area. We used the mean value.

\section{Qualitative Characteristics}

The measured horizontal wind field above the field site of HOPE showed considerable variability during the experiment. Figures 5 and 6 show examples of the streamwise wind component as well as the cross-stream wind component for different situations. Those examples are characteristic of the whole dataset. Very often, clearly visible enclosed areas of enhanced or reduced wind speed in the streamwise and/or the cross-stream wind component could be observed (Fig. 6). Those outstanding areas were often elongated and aligned with the mean background flow, which is a general feature of streaks (Adrian 2007). However, also irregular enclosed areas were observed. Both regular and irregular appeared in different sizes of about $100 \mathrm{~m}$ up to $2,000 \mathrm{~m}$. We denote these areas in the following as coherent structures. The temporal resolution is high enough to track individual structures from one timestep to the next, although their form, size, and inner structure change slightly.

A special scenario occurred during situations without background flow (calm situations). During unstable conditions, it is possible to identify areas of strong divergence and convergence in the wind field. Figure 7 shows an example of a snapshot of the wind field without 

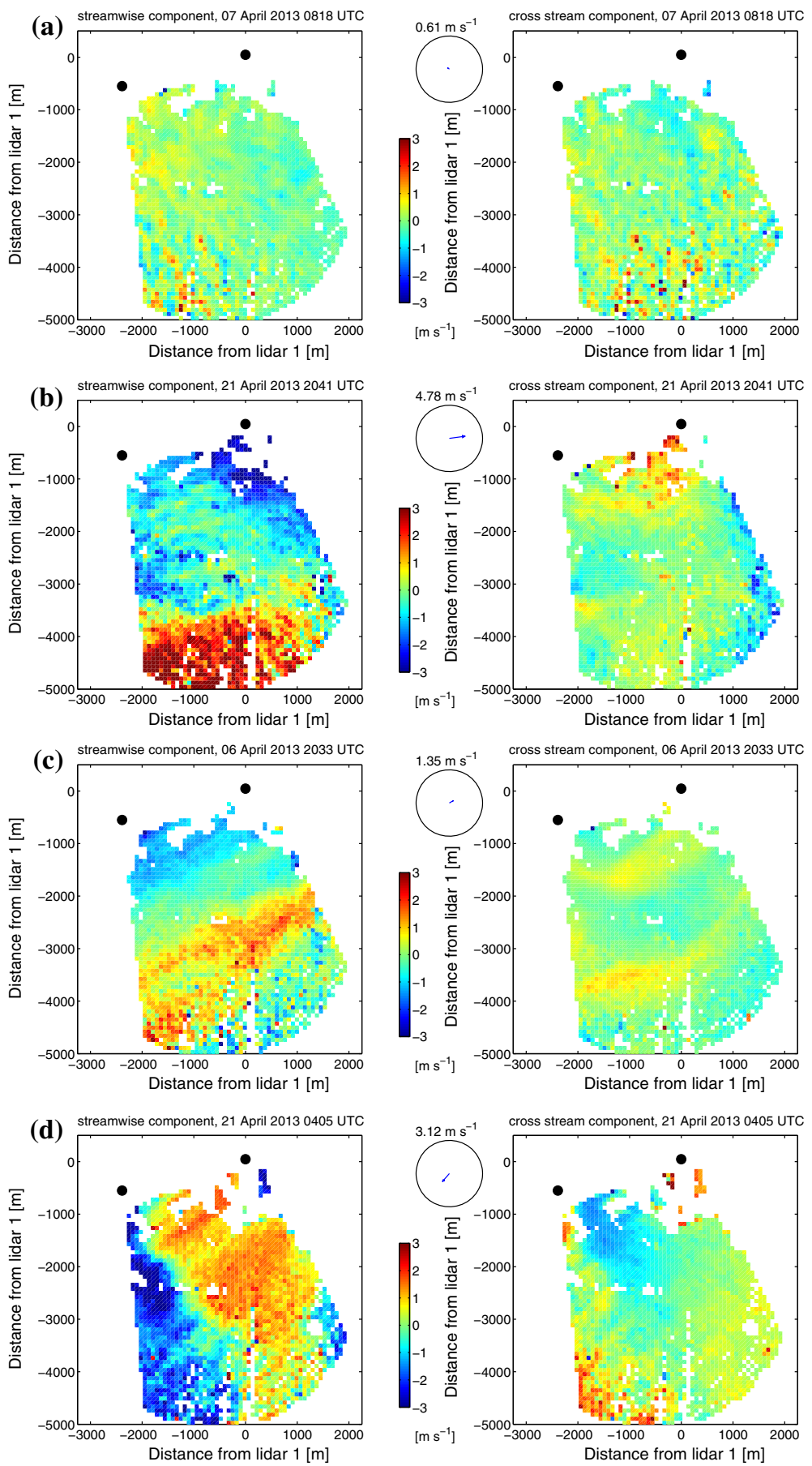

Fig. 5 Snapshots of the streamwise wind component (left) and the cross-stream wind component (right) during situations with homogeneous wind field without stratification (a) and with stratification (b), and with additional large-scale effects $(\mathbf{c}, \mathbf{d})$. The colours show the wind components $u_{1}^{\prime}($ left $)$ and $u_{2}^{\prime}($ right $)$ in $\mathrm{m} \mathrm{s}^{-1}$. The arrow indicates the wind direction and wind speed 

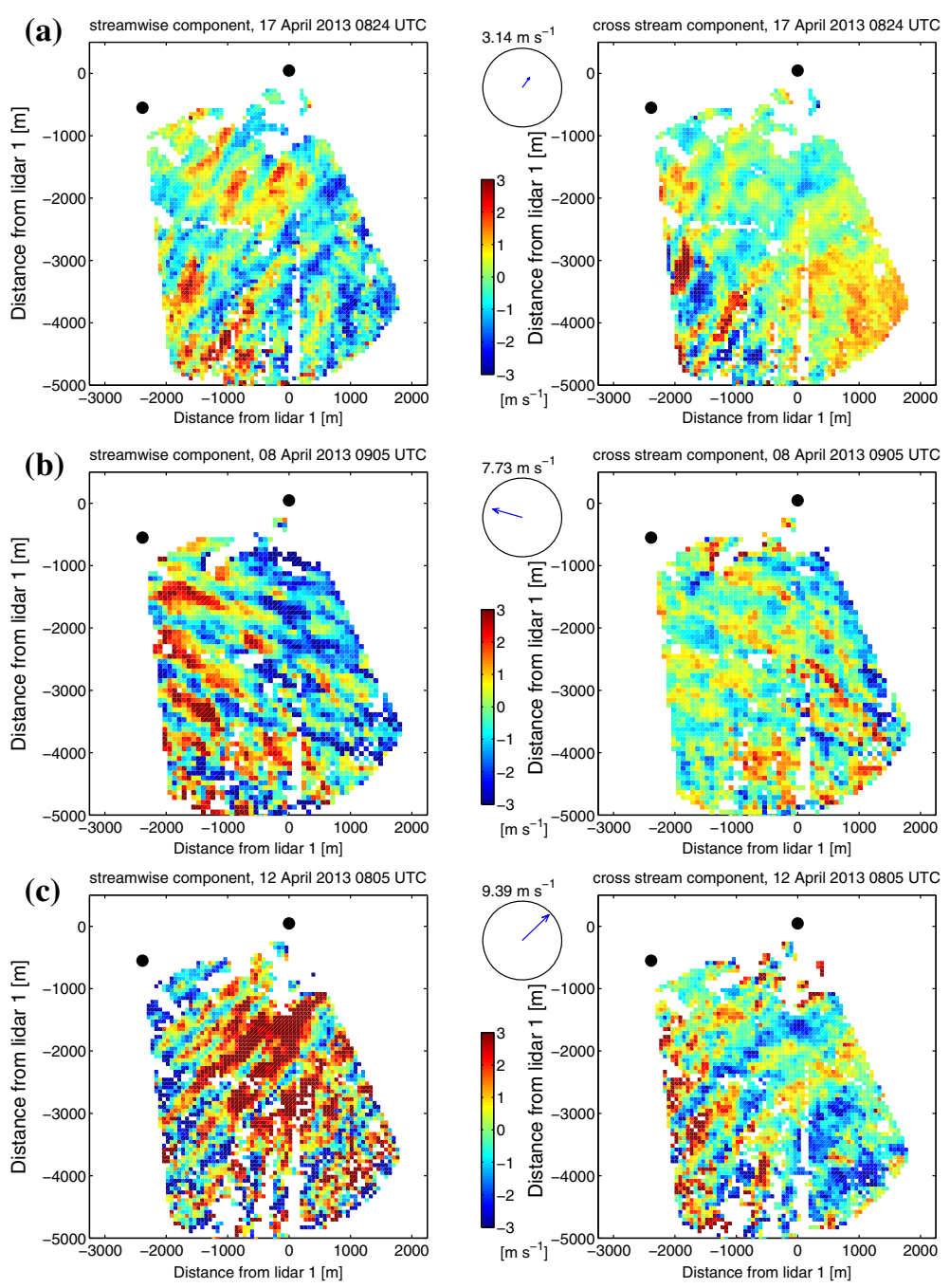

(d) streamwise component, 19 April 20131352 UTC

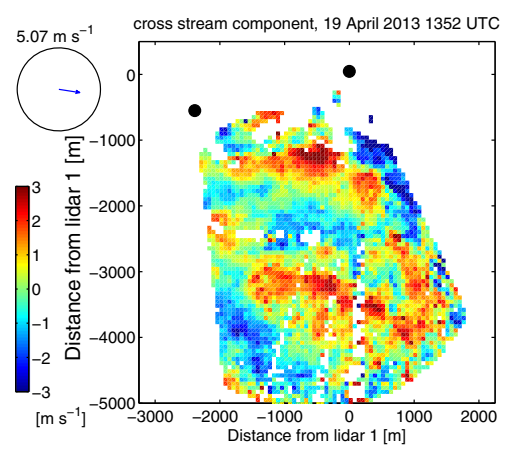

Fig. 6 Snapshots of the streamwise wind component (left) and the cross-stream wind component (right) during situations with structures. The colours show the wind components $u_{1}^{\prime}$ (left) and $u_{2}^{\prime}\left(\right.$ right) in m s${ }^{-1}$. The arrow indicates the wind direction and wind speed 

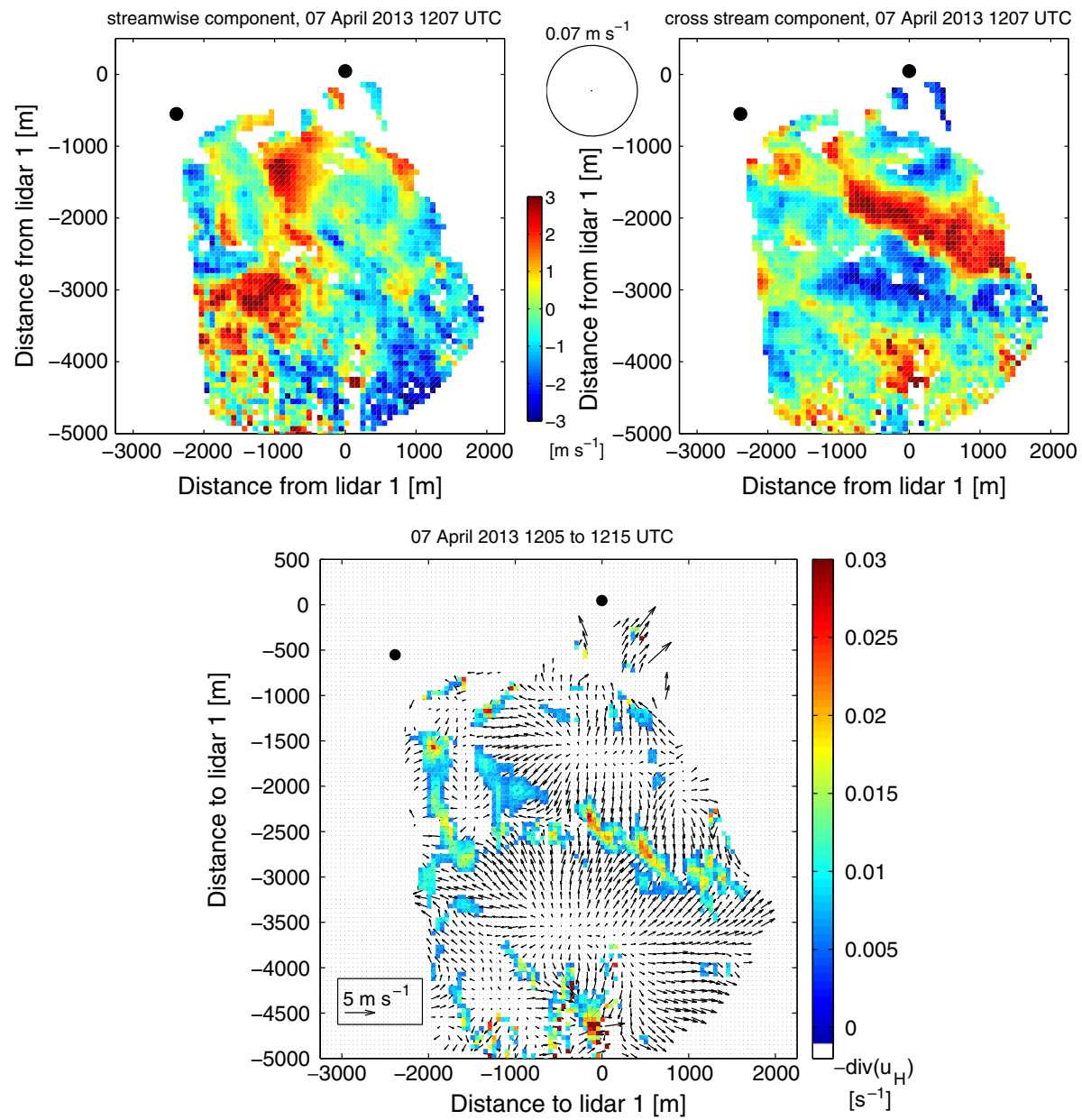

Fig. 7 Streamwise and cross-stream wind components during a situation without background flow (top) and the corresponding vector field with convergence zones (bottom)

background flow and the 10-min averaged flow field around the same situation. The coloured areas mark the zones with strong convergence (negative divergence) that indicate upward motion. The organization in the wind field in Fig. 7 shows features of open-cell convection. The pattern is quite similar to wind fields in LES with convective forcing and without background flow (see Sect. 1) and can be described as alveolar structures. The extent of these cells is on the order of $1 \mathrm{~km}$.

Although, to quote Hussain (1983), "one can usually see in flow visualization what one wants to see", a visual inspection method was used to characterize the wind field. Therefore four independent subjective analyses (by four people with meteorological backgrounds) of the wind fields were conducted for all hourly intervals and the wind fields were categorized each in one of four classes: (i) homogeneous wind fields without considerable features in the streamwise or cross-stream wind component (Fig. 5 a, b); (ii) wind fields with large-scale structures in the streamwise and/or cross-stream wind component, where large-scale implies a single dominating structure in the wind field whose extent in one direction is at least 1.5 
Table 2 Results of the four independent visual inspections of the dataset

\begin{tabular}{|c|c|c|c|c|}
\hline & \multicolumn{2}{|c|}{ Homogeneous wind fields } & \multicolumn{2}{|c|}{$\begin{array}{l}\text { Wind fields } \\
\text { with structures }\end{array}$} \\
\hline & $\begin{array}{l}\text { No. of } \\
\text { intervals }\end{array}$ & $\begin{array}{l}\text { Wind speed } \\
\left(\mathrm{m} \mathrm{s}^{-1}\right)\end{array}$ & $\begin{array}{l}\text { No. of } \\
\text { intervals }\end{array}$ & $\begin{array}{l}\text { Wind speed } \\
\left(\mathrm{m} \mathrm{s}^{-1}\right)\end{array}$ \\
\hline \multicolumn{5}{|l|}{ Night } \\
\hline \multicolumn{5}{|c|}{ Mean wind speed in all intervals $4.2 \pm 1.9 \mathrm{~m} \mathrm{~s}^{-1}$} \\
\hline Observer 1 & 118 & $3.6 \pm 1.8$ & 64 & $5.5 \pm 1.4$ \\
\hline Observer 2 & 126 & $3.8 \pm 1.7$ & 39 & $6.1 \pm 1.2$ \\
\hline Observer 3 & 113 & $2.7 \pm 1.6$ & 54 & $5.2 \pm 2.0$ \\
\hline Observer 4 & 121 & $3.6 \pm 1.6$ & 56 & $5.5 \pm 1.8$ \\
\hline Similarly identified & 78 & $3.7 \pm 1.7$ & 27 & $6.3 \pm 1.3$ \\
\hline \multicolumn{5}{|l|}{ Day } \\
\hline \multicolumn{5}{|c|}{ Mean wind speed in all intervals $4.4 \pm 2.1 \mathrm{~m} \mathrm{~s}^{-1}$} \\
\hline Observer 1 & 27 & $2.3 \pm 1.5$ & 159 & $4.9 \pm 2.0$ \\
\hline Observer 2 & 27 & $2.7 \pm 1.6$ & 151 & $4.7 \pm 2.2$ \\
\hline Observer 3 & 15 & $3.5 \pm 1.8$ & 181 & $4.5 \pm 2.2$ \\
\hline Observer 4 & 13 & $2.2 \pm 1.6$ & 169 & $4.7 \pm 2.1$ \\
\hline Similarly identified & 09 & $2.6 \pm 1.5$ & 145 & $4.8 \pm 1.1$ \\
\hline
\end{tabular}

km (Fig. 5 c and d); (iii) wind fields that contain irregular structures with an extent of several tenths of metres; and (iv) wind fields that contain periodic structures with an extent of several tenths of metres (Fig. 6, it is up to the reader whether he or she classifies the structures as periodic). From the conducted measurements it was often not possible to classify the observed structures clearly as near-surface streaks or mixed-layer rolls or, a combination of both. Therefore we use the term "structure" in general for observed organizations in the wind field.

The wind direction and the horizontal wind speed were averaged on an hourly basis from the dual Doppler data. Additionally, the time intervals between sunrise and sunset (day, 0600 to 1859 UTC) and sunset and sunrise (night, 1900 to 0559 UTC) were separated, noting that local time is UTC $+1 \mathrm{~h}$. This allows a very simple differentiation between cases with and without buoyancy forcing. Although, also during daytime, situations occur where the forcing is shear-dominated and buoyancy plays only a minor role. Effects depending on wind direction were not found.

Overall the observers classified [370, 358, 363, 360] intervals (intervals that seemed ambiguous to individual observers were not classified by them). Large-scale structures (class ii) were observed in $0-5 \%$ of the situations dependent on the observer. Such structures may indicate e.g. frontal systems or low-level jets, they are outstanding features that need a detailed analysis, which is beyond the scope of this paper.

Table 2 gives an overview of the individual visual classifications for cases without (class i) and with structures (classes iii and iv). Wind speeds for the individual configurations were approximately normally distributed. Therefore, the third and fifth column in Table 2 show the mean wind speed and its standard deviation for the subsets. The four analysis assigned $35-43 \%$ of the classified wind fields to class i (homogeneous wind field). Between 81 and $90 \%$ of these class $\mathrm{i}$ wind fields occurred during the night (Fig. 8). The class i situations during daytime showed mean wind speeds around $2.2-3.5 \mathrm{~m} \mathrm{~s}^{-1}$, which is considerably less than the mean value of $(4.4 \pm 2.1) \mathrm{m} \mathrm{s}^{-1}$ for all intervals during daytime. This implies that between 0600 UTC and 1900 UTC the situations with homogeneous wind fields were 


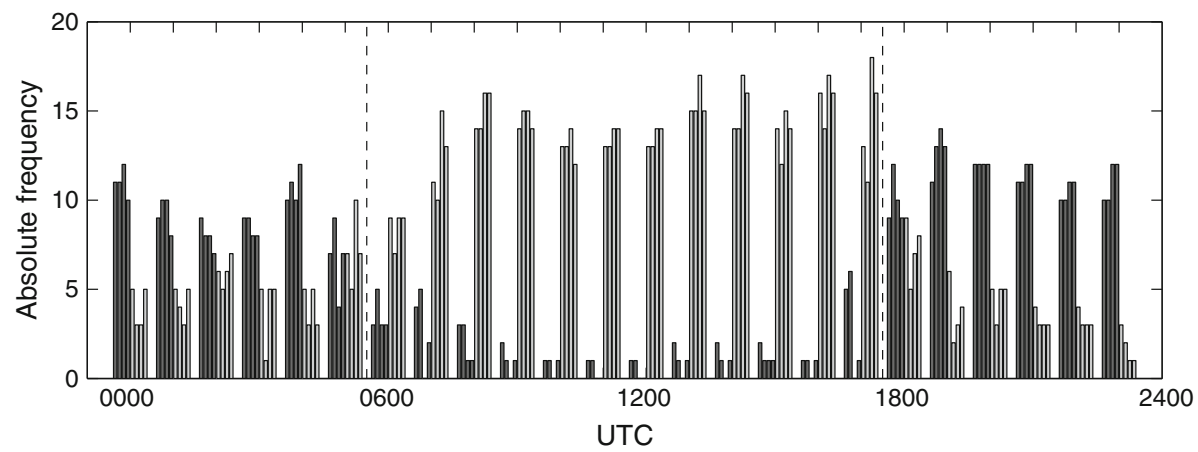

Fig. 8 Relationship between time of day (UTC) and absolute frequency of wind fields with visually classified structures (light grey) and homogeneous wind fields (dark grey)

characterized by rather low background flows. Also, during the night, the expectation value was slightly less for class i wind fields compared to the overall night value.

For 53-65\% of all hourly intervals, the wind field contained coherent structures (class iii and class iv). Concerning periodicity, the rating differed strongly between the four observers: $14,32,69$, and $77 \%$ of the cases with structures were classified as periodic (class iv); 71$77 \%$ intervals with wind fields that contain structures were observed during daytime (Fig. 8). The mean wind velocity during situations with structures was between 4.5 and $4.9 \mathrm{~m} \mathrm{~s}^{-1}$ and is, therefore, slightly enhanced compared to the overall value. During nighttime, this effect was much more pronounced. The mean wind speed with values between 5.2 and $6.1 \mathrm{~m} \mathrm{~s}^{-1}$ was here considerably higher than the overall value of $4.2 \mathrm{~m} \mathrm{~s}^{-1}$, implying that, during the night, stronger flow seemed to trigger the structures. All observers noticed a trend to higher periodicity during the changeover from night to day and vice versa. Furthermore, periodicity was observed rarely during the night, where only $14-22 \%$ of all class iv situations were identified.

In summary, structures seemed to be a feature of wind fields characterized by stronger background flow and occurred preferably between sunrise and sunset. It was possible to observe changeover processes between situations with and without structures. Typical features of streaks (elongation, alignment with wind direction) could be observed, although the often described periodicity was difficult to specify, especially in the relatively small section of the wind field that could be obtained by the instruments. In some cases, spatial periodicity was clearly visible and it was possible to observe the changeover between periodic and non-periodic patterns. The subjective analysis described here is quite simple and does not reveal details of the generation processes of structures, but gives indicators based on two-dimensional measurements, which are quite rare to date.

\section{Coherence Length Characteristics}

To provide a quantitative analysis of coherence, the integral length scales of the streamwise $\left(u_{1}\right)$ and the cross-stream $\left(u_{2}\right)$ wind components in the streamwise $(x)$ and the cross-stream (y) directions, were evaluated. Ratios of these quantities were used to classify isotropy and additional maxima in the autocorrelation function to characterize periodicity. Although this approach allows conclusions concerning the coherence of the wind field, it is not suitable for characterizing individual structures. Because of the variability of the wind field between 

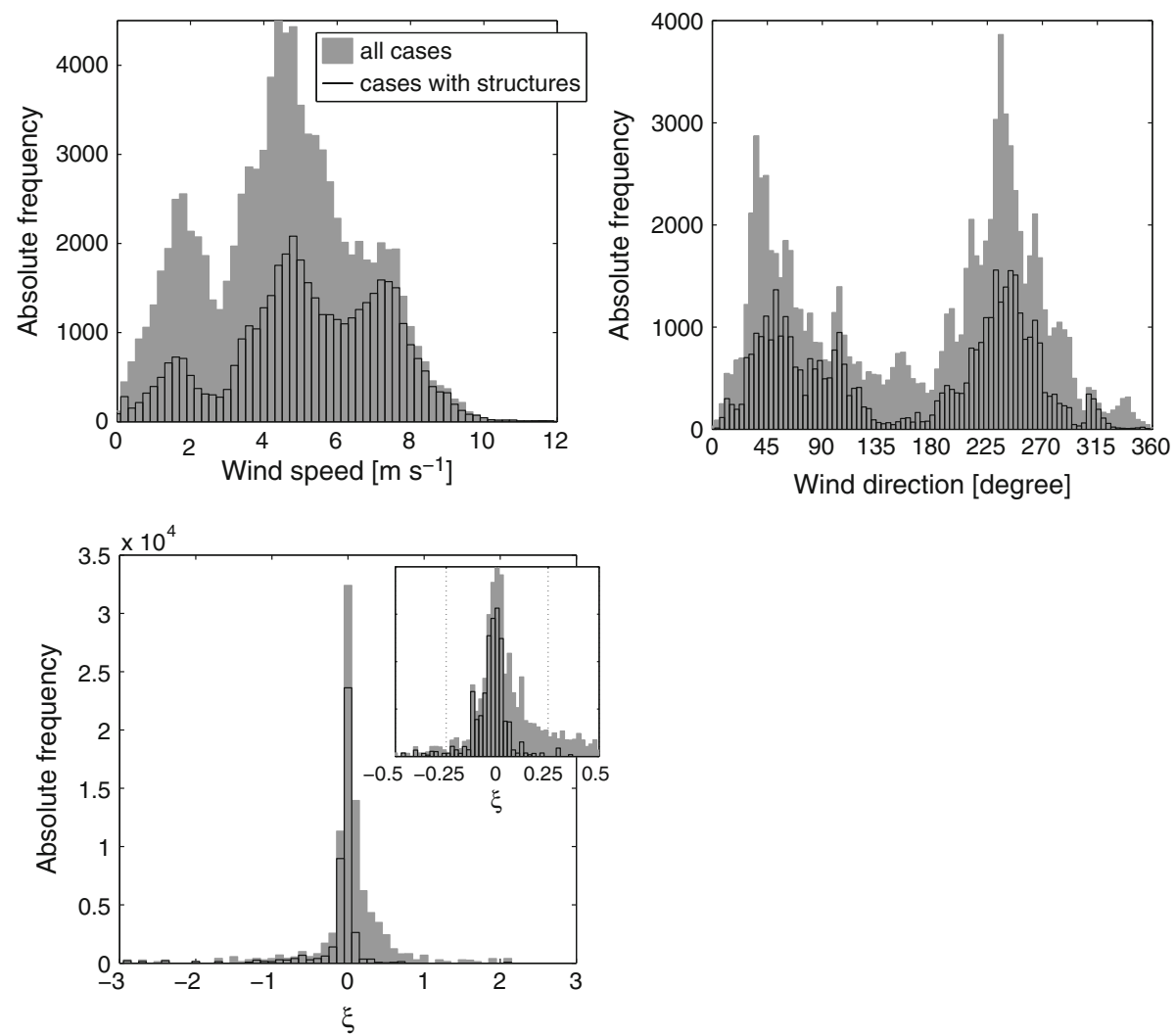

Fig. 9 Distribution of the wind speed (left), the wind direction (middle) as well as the stability parameter $\xi$ (right) for all the considered intervals. The grey bars show all cases, the black bars mark the cases with structures

adjacent timesteps, each wind field that contains more than 1,500 data points is considered for statistical interpretation even though adjacent wind fields are not independent. In total, 93,335 individual time intervals were available, corresponding to more than $300 \mathrm{~h}$. Figure 9 gives an overview of the prevailing wind conditions as well as the distribution of the stability parameter $\xi$. The wind fields that were classified to contain structures by all four observers at once in the previous section were marked using black edging. It is obvious that the largescale forcing during the selected HOPE days favoured low wind-speed situations with wind speeds between 1 to $2 \mathrm{~m} \mathrm{~s}^{-1}$. The wind fields with structures preferred higher wind speeds. Concerning wind direction no differences are obvious between the whole dataset and the cases with structures. For $\xi<0$ about $70 \%$ of the wind fields were classified to contain structures by all four observers at once, whereas only $6 \%$ were classified as homogeneous ( $24 \%$ were classified ambiguously). In this region the behaviour is dominated by wind fields with structures. For $\xi>0.2$ about $62 \%$ of the wind fields were classified as homogeneous by all four observers, whereas only $5 \%$ contained subjectively structures (again $33 \%$ were classified ambiguously). Here the behaviour is dominated by homogeneous wind fields. The regime between $\xi>0$ and $\xi<0.2$ contains cases with stronger shear but no buoyancy forcing. Here $38 \%$ of the cases contained structures corresponding to all four observers, 
$16 \%$ of the wind fields were classified as homogeneous but the utmost part was classified ambiguously.

Table 3 shows all considered variables for the eight situations in Figs. 5 and 6. These examples demonstrate the variability of the integral length scales as well as the related anisotropy. From these eight situations, it is not possible to draw a conclusion concerning relationships of the integral length scales or the anisotropy to the background flow or the stability of the atmosphere. From visual inspection, the cases $6 \mathrm{a}-\mathrm{c}$ show streak-like periodic structures. For these three cases, the autocorrelation function in the cross-stream direction shows additional maxima. However, also the homogeneous wind fields in Fig. 5a-c show, at least in the $u_{2} y$ component, some kind of periodicity as well. Therefore, the occurrence of additional maxima appears to be a necessary but not sufficient condition for streaky structures.

To achieve more reliable results, it is necessary to draw conclusions from the complete dataset. The integral length scales of the streamwise wind component as well as the crossstream component could be determined (i.e., a zero crossing of the autocovariance function was present) in around $90 \%$ of all cases. Figure 10 shows the distributions for all four composites. Due to the lidar resolution and the associated grid resolution, scales below $100 \mathrm{~m}$ were not detected. All four distributions follow an asymmetric course, with most frequent values around $[350,250,400,250] \mathrm{m}$ for $\left[l_{u_{1} x}, l_{u_{1} y}, l_{u_{2} x}, l_{u_{2} y}\right]$. For both wind components, the most frequent length scales in the cross-stream direction are smaller than those in the streamwise direction. Associated, the distributions of the cross-stream length scales show a steeper increase. All four distributions show an equivalent decrease up to values of approximately $1,200 \mathrm{~m}$. The wind fields that have been classified by all four observers at once in the previous section to contain structures show a trend to shorter integral length scales especially for the cross-stream wind components, although they follow the same distribution.

Figure 11 shows the distributions of the ratios $l_{u_{i} x} / l_{u_{i} y}$. For $u_{1}, 69 \%$ of the ratios and for $u_{2}, 71 \%$ of the ratios, respectively, show values $>1$ i.e., more than two-thirds show an elongation of the eddies in the flow direction. The majority of the situations (55 and $56 \%$, respectively) show a ratio between $2 / 3$ and $3 / 2$ i.e., represent nearly isotropic wind fields. However, also strong anisotropy can be observed; $16 \%$ of the $u_{1}$ ratios and $18 \%$ of the $u_{2}$ ratios, respectively, show values $>2,3 \%$ with ratios $>3$. Ratios of $l_{u_{i} x} / l_{u_{i} y}>2$ show a considerably larger coherence in the streamwise direction than in the cross-stream direction. A high ratio may be related to streaky structures. Only about $2 \%$ of the calculated ratios for the streamwise wind component, $1 \%$ for the cross-stream wind component respectively, are $<0.5$ i.e., show a considerably larger integral length scale in the cross-stream than in the streamwise direction. A ratio of $<0.5$ may be an indication of atmospheric waves. If only cases are considered that contain structures corresponding to all four observers from the previous section, the distributions shift to higher ratios for both, the streamwise and cross-stream wind directions.

To illustrate the dependencies on the stability parameter $\xi$ and the wind speed, a boxplot scheme is used in the following. For stability, the data were collected at 0.05 intervals for $\xi$, and for wind speed in $1 \mathrm{~m} \mathrm{~s}^{-1}$ intervals. Boxes were only drawn if more than 50 values could be associated to the interval. In each box, the central mark is the median and the edges are the 25th $\left(q_{1}\right)$ and 75th $\left(q_{3}\right)$ percentiles. Outliers were defined as values beyond the interval $\left[q_{1}-1.5\left(q_{3}-q_{1}\right) q_{3}+1.5\left(q_{3}-q_{1}\right)\right]$. To ascertain that the integral length scales are related to the respective parameter, the rank correlation coefficient after Spearman $R$ was calculated and a $t$ test was used to identify significance. Thereby, the hypothesis of independence could be rejected with a probability of an error of $2 \%$ if $t=+R \sqrt{n-2}\left(\sqrt{1-R^{2}}\right)^{-1}>2.33$, where $n$ is the number of time frames. 


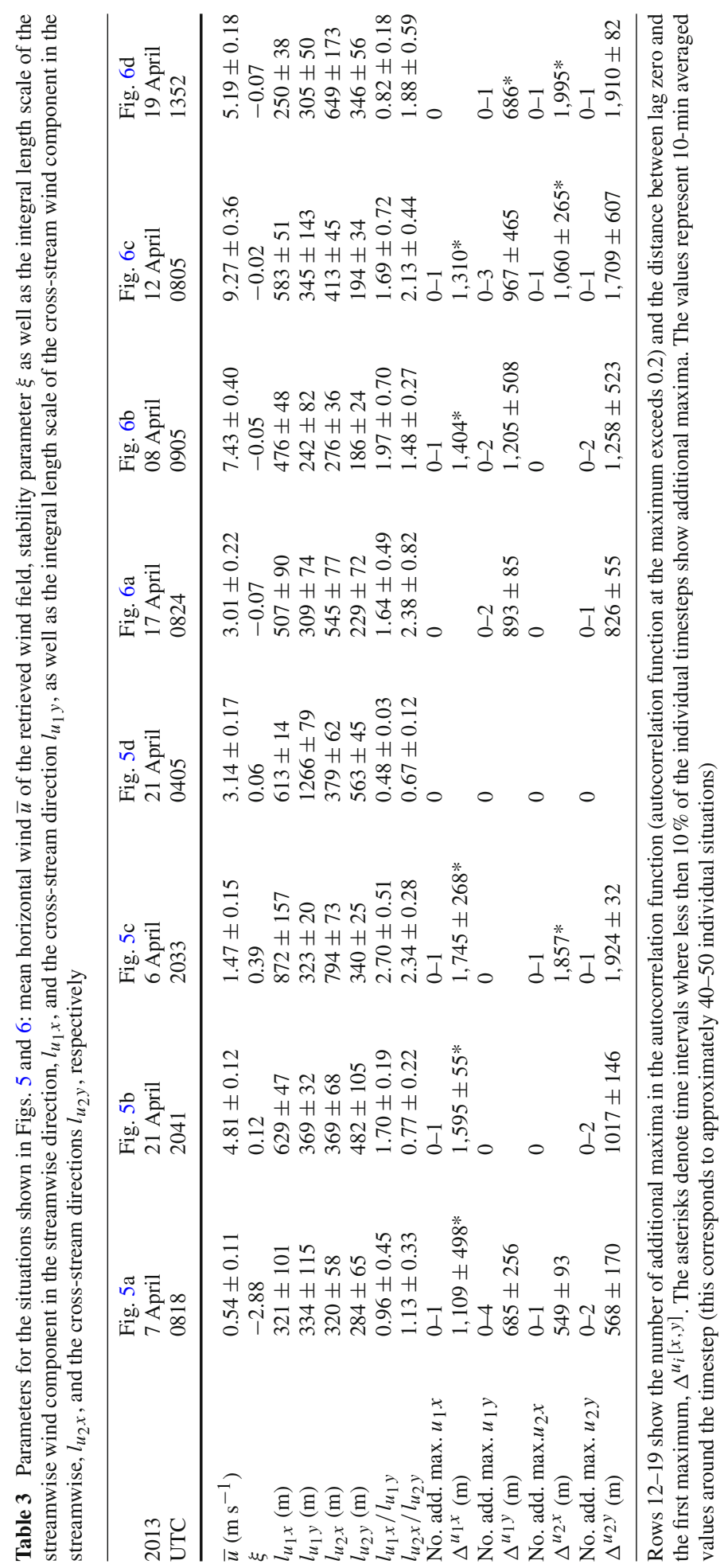



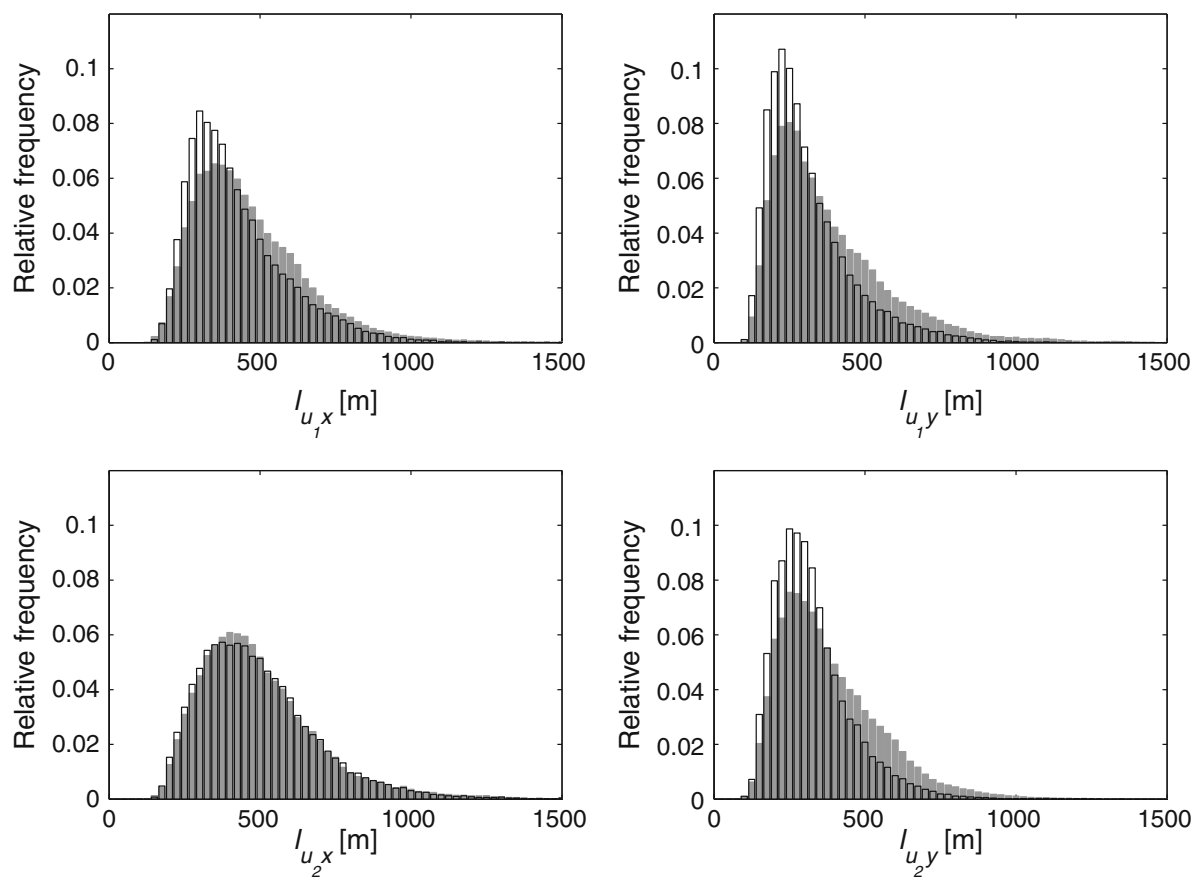

Fig. 10 Distribution of the integral length scales for the streamwise component (top) in the streamwise direction (left), in the cross-stream direction (right), and the cross-stream component (bottom) in the streamwise direction (left) as well as in the cross-stream direction (right). The grey boxes indicate all cases, the black edging shows only cases that contain structures
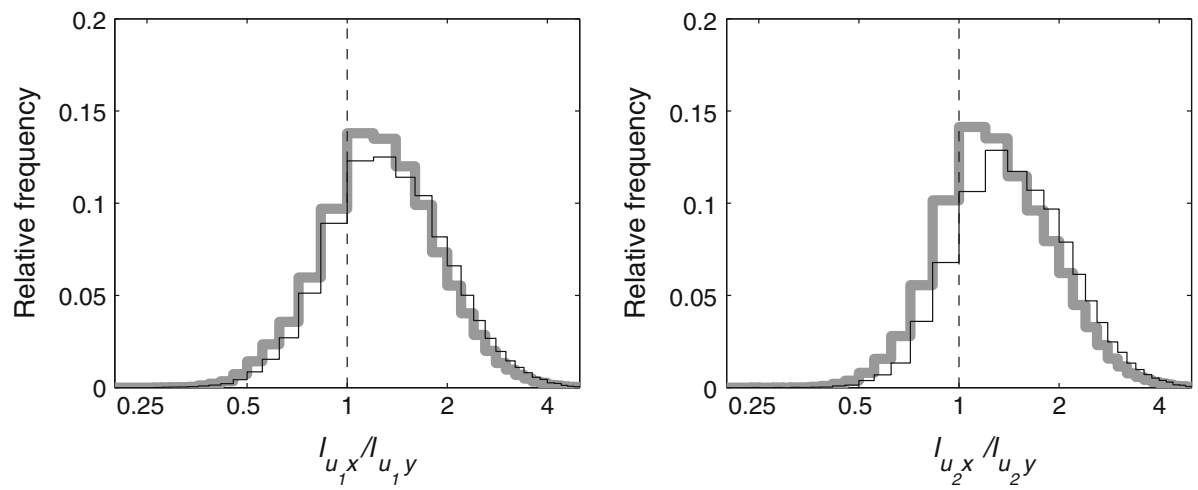

Fig. 11 Distribution of anisotropy of the streamwise wind component (left) and the cross-stream component (right). The grey line indicates the distribution for all cases, the black only for cases classified to contain structures

Figure 12 shows the relationship between the integral length scales and the stability parameter $\xi$. For evaluation only data with $\xi \in[-0.50 .5]$ were considered, which corresponds to over $84 \%$ of the values and ensures that the high amount of data for low $|\xi|$ is not too much restrained by very few high values. $l_{u_{1} x}, l_{u_{1} y}$, and $l_{u_{2} x}$ show a correlation with $R=$ $0.29,0.36$, and 0.35 ( $t$ is 80,102 , and 101 i.e., the relation is highly significant). All three 

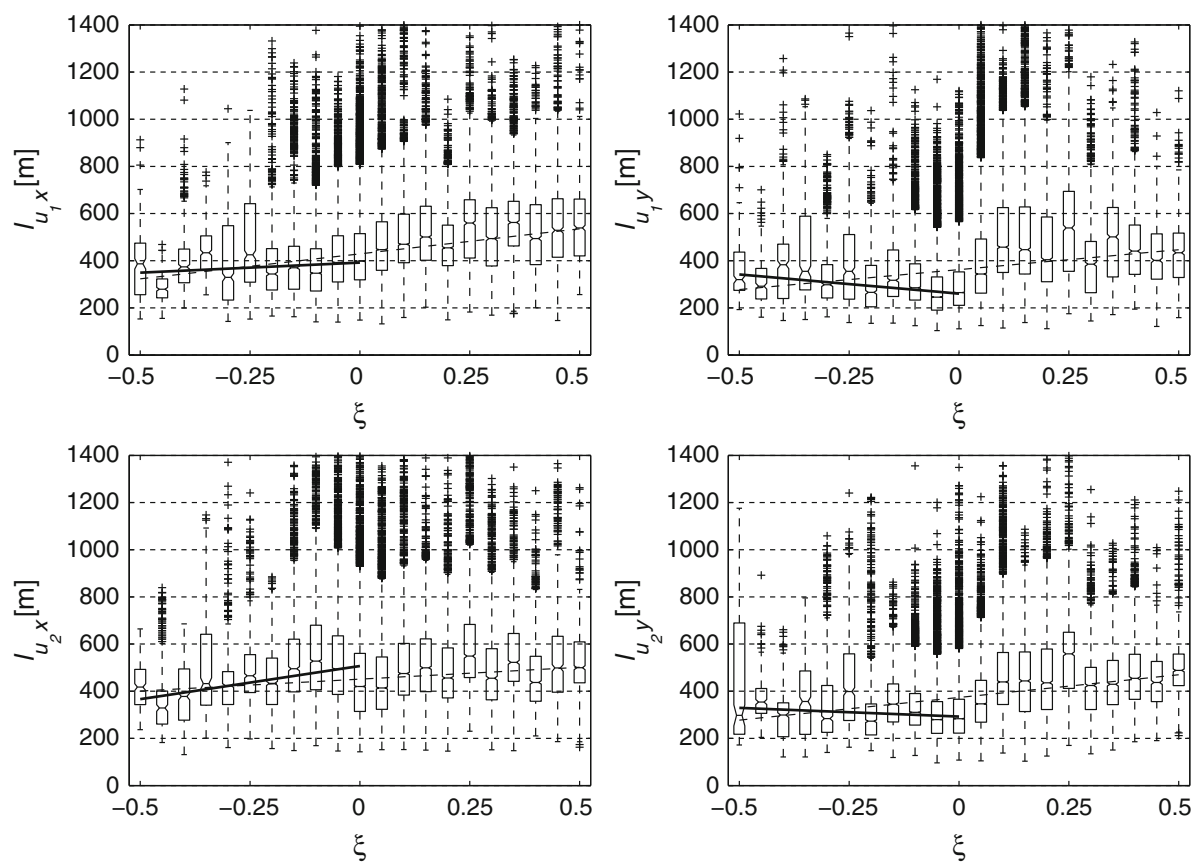

Fig. 12 Relationship between stability and integral length scales for the streamwise wind component (top) and the cross-stream wind component (bottom) in the stream direction (left) as well as in the cross-stream direction (right). The dashed line marks the overall trend, the strong line indicates the trend for wind fields with streaky structures, the boxplot scheme is described in the text, the crosses represent the outliers

show an analogous behaviour of the median values with a slight increase with increasing stability. The correlation coefficient between $\xi$ and $l_{u_{2} x}$ of $0.0(t=0.7)$ does not indicate a monotonic relationship. For the streamwise velocity component, the integral length scales for both directions increase quite similarly i.e., the stability has hardly any effect on anisotropy ( $R=-0.13, t=-33$ ). For the cross-stream wind component, the different behaviour of $l_{u_{2} x}$ leads to an effect on anisotropy with $R=-0.34(t=-89)$. With increasing stability, $l_{u_{2} x} / l_{u_{2} y}$ decreases slightly from 1.5 to 1 . Using the classification from the previous section, the behaviour is dominated by cases with structures for $\xi<0$ (see Fig. 9). Going from $\xi=-0.5$, where the shear production and the buoyant production term are approximately equal (for convective conditions, Stull 1988), to $\xi=0$ where the shear dominates, the velocity components evaluated in the streamwise directions show a slight tendency to increase whereas the velocity components evaluated in the cross-stream direction tend to decrease. A more dominating shear seems to be connected to higher anisotropy and narrower structures.

Newsom et al. (2008) used a similar approach to obtain integral length scales. Their integral length scales were in the same order of magnitude, but they were not able to identify a trend concerning changing stability. The outstanding maximum of $l_{u_{1} x}$ for neutral conditions found by Newsom et al. (2008) and the high aspect ratios (more than two-thirds are higher than $3.5)$ could not be confirmed. The reason might be the different surface conditions that might favoured long correlation lengths during the JU2003 experiment and destroyed the correlation during HOPE. Huang and Bou-Zeid (2013) also performed a two-point autocorrelation analysis to characterize coherent structures based on LES data under stable situations. They found an increase of the integral length scale of the streamwise velocity component with 
stability, which is in accordance with the results found in this study. It should be mentioned here, that the behaviour of the vertical wind component (which is not considered here) typically shows a reversed behaviour. With increasing stability the integral length scales decrease (Salesky et al. 2013; Huang and Bou-Zeid 2013).

The relationship between the wind speed and the integral length scales was analyzed separately for different stability regimes. At once, this procedure allows a conclusion for wind fields with structures that dominate the behaviour for $\xi<0$, and homogeneous wind fields that dominate the behaviour for $\xi>0.2$.

For strongly stable cases with $\xi>0.2$, correlation coefficients of $R=[0.16,0.25,0.18$, $0.25]$ between the wind speed and $\left[l_{u_{1} x}, l_{u_{1} y}, l_{u_{2} x}, l_{u_{2} y}\right]$ are found, which indicate a trend of larger length scales for higher wind speeds. The eddies show a quite isotropic behaviour with 50th percentile of the ratios $l_{u_{i} x} / l_{u_{i} y}$ at 1.19 for $i=1$, and 1.08 for $i=2$ respectively.

During weakly stable conditions $(0<\xi<0.2)$, this behaviour changes considerably. Now, all four combinations reveal correlation coefficients between $R=-0.18$ and -0.27 . So they show an integral length scale decrease with increasing wind speed i.e., in this regime the higher wind speed and mostly corresponding higher shear destroys the correlation. The wind fields are mostly isotropic and the wind speed has a slight effect on the ratio $l_{u_{1} x} / l_{u_{1} y}$ with $R=0.19(t=30)$ and $l_{u_{2} x} / l_{u_{2} y}$ with $R=0.07(t=12)$. The 50th percentile of the ratio lies at 1.22 for $i=1$, and 1.18 for $i=2$ respectively, and therefore somewhat higher than for the strongly stable cases.

The wind fields with structures were generally observed during unstable situations with $\xi<0$. Figure 13 shows the relationship between the wind speed and the integral length scales for those situations. The integral length scales for both wind components in the streamwise direction show a positive correlation with $R=0.19(t=34)$ between wind speed and $l_{u_{1} x}$ and $R=0.08(t=14)$ between wind speed and $l_{u_{2} x}$. On the other hand, in the crossstream direction a negative correlation can be observed with $R=-0.27(t=51)$ between wind speed and $l_{u_{1} y}$, and $R=-0.22(t=42)$ for $l_{u_{2} y}$ respectively. This means that with increasing wind speed, the eddies seem to elongate in the streamwise direction and become narrow in the cross-stream direction. This behaviour is also reflected in the ratios $l_{u_{i} x} / l_{u_{i} y}$ that show correlation coefficients of 0.39 for $i=1$, and in 0.27 for $i=2$ respectively. For both wind directions an increase of the median values for $l_{u_{i} x} / l_{u_{i} y}$ from 1 for calm situations to 2 for wind speeds $\geq 8 \mathrm{~m} \mathrm{~s}^{-1}$ is observable. For wind speeds $>6 \mathrm{~m} \mathrm{~s}^{-1}$ the 95 th percentile of $l_{u_{i} x} / l_{u_{i} y}$ exceeds 3 for both $i=1$ and $i=2$. Such high ratios are not achieved for the stable and weakly stable cases.

In summary, for stable and unstable conditions, isotropy was reached during calm situations. During unstable conditions, the flow was able to elongate the eddies in the mean wind direction, whereas during stable conditions, this effect was restrained. This result is in contradiction to the findings of Newsom et al. (2008) who described strong anisotropy in all stability regimes for the streamwise wind component. Also Huang and Bou-Zeid (2013) observed an anisotropic behaviour during stable conditions. It should be mentioned, that also individual cases in this study differ from the median behaviour.

Additional maxima in the autocorrelation function are found in $8 \%$ of the cases for $l_{u_{1} x}, 16 \%$ for $l_{u_{1} y}, 11 \%$ for $l_{u_{2} x}$, and $12 \%$ for $l_{u_{2} y}$. This means that periodicity on scales smaller than $2,000 \mathrm{~m}$ is considerably more frequent in the cross-stream direction than in the streamwise direction for the streamwise wind component and similar frequent for the crossstream wind component. No clear trend was found in the relationship between periodicity and the background wind speed or $\xi$.

The distances between lag zero and the lag at the next maximum with $r_{u_{i}}>0.2$ varies between 300 and 2,000 $\mathrm{m}$ (the latter was used as the upper limit). The absolute frequency of 

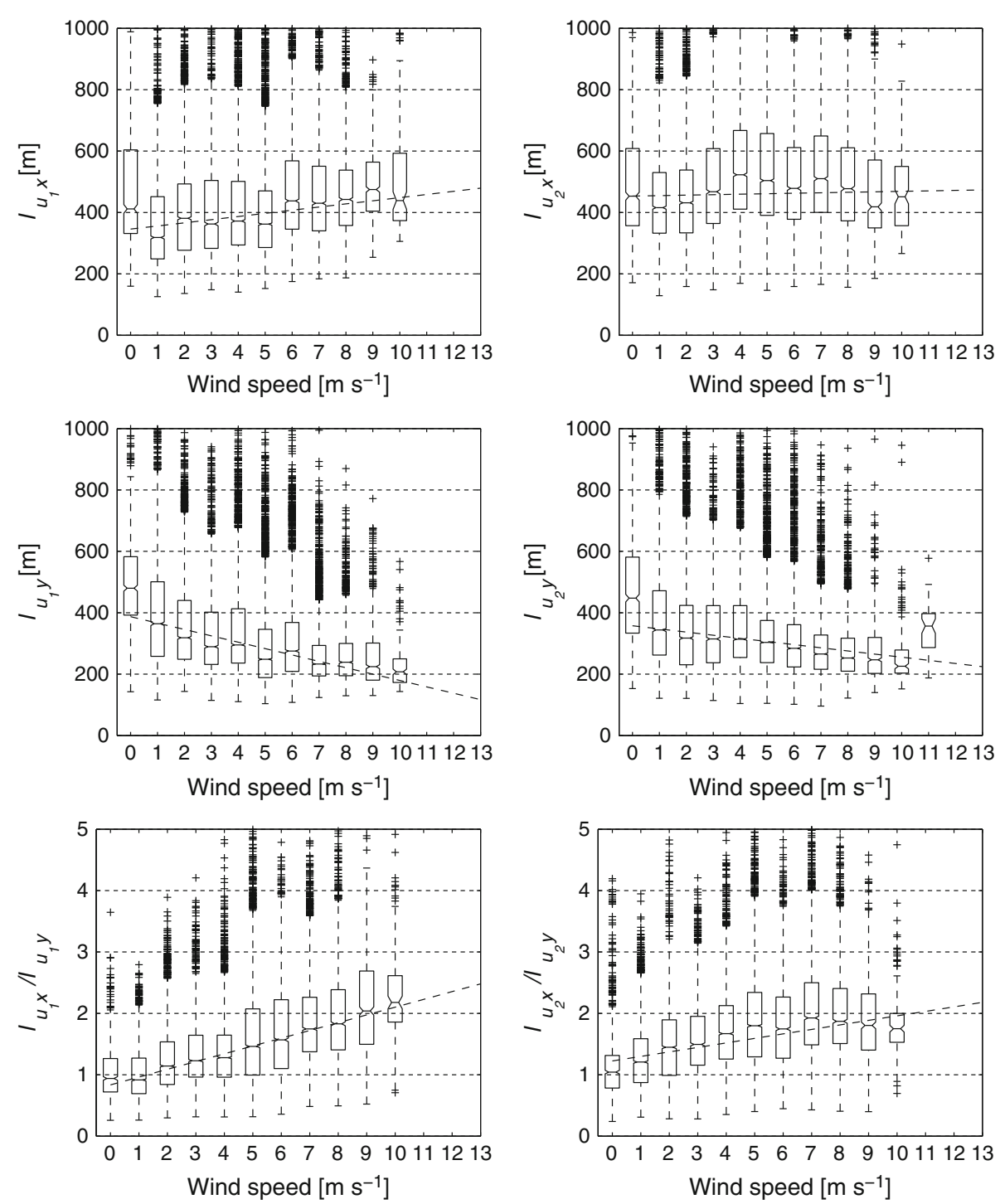

Fig. 13 Relationship between the background flow and integral length scales for situations with $\xi<0$ for the streamwise wind component (left) and the cross-stream wind component (right)

occurrence is equally distributed. The averaged values are $\Delta^{u_{1} x}=1.088 \pm 712 \mathrm{~m} \Delta^{u_{1} y}=$ $1.257 \pm 607 \mathrm{~m}, \Delta^{u_{2} x}=882 \pm 790 \mathrm{~m}$ and $\Delta^{u_{2} y}=1.344 \pm 591 \mathrm{~m}$. These values are on the order of the boundary-layer height and provide no general information about the distance between streaks.

\section{Summary}

Coherent structures are a feature of turbulence in the atmospheric surface layer. An advanced method to observe these structures is the application of a dual Doppler lidar set-up in horizon- 
tal scanning mode. During the HOPE field campaign in spring 2013 near Jülich, Germany, this type of set-up was realized. Using a retrieval technique, it was possible to visualize the streamwise and the cross-stream wind components in an area of approximately $12 \mathrm{~km}^{2}$ for almost $300 \mathrm{~h}$ with a temporal resolution of about $12 \mathrm{~s}$ and a spatial resolution of only $70 \mathrm{~m}$.

For this study, the dataset was analyzed for coherent structures in two ways: a subjective visual inspection of film clips created for all measurement days and an objective method using the two-dimensional autocorrelation function. The subjective method revealed that the wind fields exhibit different kinds of characteristic patterns: homogeneous wind fields, wind fields with structures that seem to be only partly captured by the lidar overlap area, and wind fields with clearly visible bounded areas of enhanced or reduced speed. These areas were considered as coherent structures, and were often elongated and aligned in the wind direction, which is a typical feature of near-surface streaks described in the literature. During several situations, a periodic behaviour was obvious. Transitions from situations without to situations with streak-like structures were visible as well as changeovers from smaller to larger structures, or higher periodicity to less. During calm situations with strong buoyancy forcing, the wind field exhibited clear convergence and divergence areas. The horizontal divergence field during such situations showed structures quite similar to open-cell convection with diameters of about $1 \mathrm{~km}$. The subjective analysis revealed that the occurrence of the structures seemed to be linked to forcing, either by shear or by buoyancy.

The dataset was analyzed objectively by using a two-dimensional autocorrelation function. Integral length scales were calculated for the streamwise and cross-stream wind components, both in and across the mean wind direction. This technique allowed the calculation of all four combinations without the application of Taylor's hypothesis. Compared to the widely used wavelet technique that deals with individual structures, this approach delivers characteristic length scales for the whole area. These length scales combine characteristics of several structures of different sizes in the wind field. Therefore, a comparison of results from the wavelet technique is not meaningful.

The length scales in the streamwise and cross-stream directions varied strongly and ranged from 100 to $1,200 \mathrm{~m}$, showing a strong increase in frequency up to $400 \mathrm{~m}$ for the streamwise direction and $250 \mathrm{~m}$ for the cross-stream direction, respectively, and a slow decrease afterwards. The ratio between the integral length scale in the streamwise direction to that in the cross-stream direction was for $55 \%$ of all cases between 0.67 and 1.5, i.e. the wind fields are approximately isotropic. More than $16 \%$ showed a ratio $l_{u_{i} x} / l_{u_{i} y}>2$, i.e., the eddies were clearly elongated in the streamwise direction.

The integral length scales were smaller during unstable situations with $\xi<0$ compared to stable situations. During unstable conditions, which were dominated by wind fields with structures, a slight decreasing trend of $l_{u_{i} y}$ with increasing $\xi$ for $-0.5<\xi<0$ was observed, which indicates narrower structures with increasing shear contribution. Also during unstable situations, a relationship between increasing background wind speed and increasing integral length scales in the streamwise direction and decreasing integral length scales in the crossstream direction became obvious. This implies that eddies become elongated in the mean wind direction and become narrower. Consequently, the anisotropy increased with increasing wind speed from 1 (isotropy) to values of 2-3. For near-neutral situations, the integral length scales decreased with increasing wind speed, and the ratio $l_{u_{i} x} / l_{u_{i} y}$ was $\approx 1$. For stable situations, a slight increase of all integral length scales with increasing wind speed was observed. Again, the eddies were isotropic in the median. Additional maxima in the autocorrelation function, which were an indicator of periodicity, were found in up to $16 \%$ of the cases. Periodicity was observed more frequently during daytime and in the cross-stream direction, but no simple relationship to wind speed or stability could be observed. 
The study revealed different kinds of structures. Simplified relationships between the occurrence of the structures, the background flow, and atmospheric stability were obvious. However, a comprehensive analysis of the corresponding length scales exhibited a high variability of turbulence behaviour. The background wind speed and the stability may determine the median behaviour but the individual situations can vary strongly for the same wind field and stability configuration. Individual snapshots of shorter time intervals are typically not representative.

Acknowledgments We want to thank the whole KITcube team for the assembly and the operation of the instruments, especially Dr. Ulrich Corsmeier and Dr. Norbert Kalthoff for their encouragement in many aspects of the project, and our students Christina Graf and Julia Kosch for many hours in front of wind field video clips. The two energy balance stations from Jülich were founded by the TERENO project, the one from Cologne by the DFG-Sonderforschungsbereich TR32 "Patterns in Soil-Vegetation-Atmosphere-Systems: Monitoring, Modelling and Data Assimilation", the personnel in charge for all three energy balance stations was funded by SFB TR32. We thank Alexander Graf, Marius Schmidt and Jan Schween for providing us with the energy balance data. The whole work was embedded in a young investigator group founded by the KIT in the framework of the German Excellence Initiative.

Open Access This article is distributed under the terms of the Creative Commons Attribution License which permits any use, distribution, and reproduction in any medium, provided the original author(s) and the source are credited.

\section{References}

Adrian R (2007) Hairpin vortex organization in wall turbulence. Phys Fluids 19:041301-1-041301-16

Barthlott C, Drobinski P, Fresquet C, Dubos T, Pietras C (2007) Long-term study of coherent structures in the atmospheric surface layer. Boundary-Layer Meteorol 125:1-24

Browning K, Wexler R (1968) The determination of kinematic properties of a wind field using Doppler radar. J Appl Meteorol 7:105-113

Drobinski P, Foster R (2003) On the origin of near-surface streaks in the neutrally-stratified planetary boundary layer. Boundary-Layer Meteorol 108:247-256

Drobinski P, Brown R, Flamant P, Pelon J (1998) Evidence of organized large eddies by ground-based Doppler lidar, sonic anemometer and sodar. Boundary-Layer Meteorol 88:343-361

Drobinski P, Carlotti P, Newsom R, Banta R, Foster R, Redelsperger JL (2004) The structure of the near-neutral atmospheric surface layer. J Atmos Sci 61:699-714

Etling D, Brown R (1993) Roll vortices in the planetary boundary layer: a review. Boundary-Layer Meteorol 65(3):215-248

Feingold G, Koren I, Wang H, Xue H, Brewer A (2012) Precipitation-generated oscillations in open cellular cloud fields. Nature 466:849-852

Fresquet C, Dupont S, Drobinski P, Dubos T, Barthlott C (2009) Impact of terrain heterogeneity on coherent structure properties: Numerical approach. Boundary-Layer Meteorol 133:71-92

Hartmann J, Kottmeier C, Raasch S (1997) Roll vortices and boundary-layer development during a cold air outbreak. Boundary-Layer Meteorol 84:45-65

Hasel M, Kottmeier C, Corsmeier U, Wieser A (2005) Airborn measurements of turbulent trace gas fluxes and analysis of eddy structure in the convective boundary layer over complex terrain. Atmos Res 74:381-402

Hellsten A, Zilitinkevich S (2013) Role of convective structures and background turbulence in the dry convective boundary layer. Boundary-Layer Meteorol 149(3):323-353

Huang J, Bou-Zeid E (2013) Turbulence and vertical fluxes in the stable atmospheric boundary layer. Part I: a large-eddy simulation study. J Atmos Sci 70(6):1513-1527

Hussain A (1983) Coherent structures-reality and myth. Phys Fluids 26(10):2816-2850

Inagaki A, Kanda M (2010) Organized structure of active turbulence over an array of cubes within the logarithmic layer of atmospheric flow. Boundary-Layer Meteorol 135:209-228

Iwai H (2008) Dual-Doppler lidar observation of horizontal convective rolls and near-surface streaks. Geophys Res Lett 35(14):L14808 
Kalthoff N, Adler B, Wieser A, Kohler M, Träumner K, Handwerker J, Corsmeier U, Khodayar S, Lambert D, Kopmann A, Kunka N, Dick G, Ramatschi M, Wickert J, Kottmeier C (2013) Kitcube-a mobile observation platform for convection studies deployed during HyMeX. Meteorol Z 22(6):633-647

Kim SW, Park SU (2003) Coherent structures near the surface in a strongly sheared convective boundary layer generated by large-eddy simulation. Boundary-Layer Meteorol 106:35-60

Lenschow D, Stankov B (1986) Length scales in the convective boundary layer. J Atmos Sci 43(12):1198-1209

Mauder M, Cuntz M, Drüe C, Graf A, Rebmann C, Schmid HP, Schmidt M, Steinbrecher R (2013) A strategy for quality and uncertainty assessment of long-term eddy-covariance measurements. Agric For Meteorol 169(0):122-135

Moeng CH, Sullivan P (1994) A comparison of shear- and buoyancy-driven planetary boundary layer flows. J Atmos Sci 51(7):999-1022

Newsom R, Calhoun R, Ligon D, Allwine J (2008) Linearly organized turbulence structures observed over a suburban area by dual-Doppler lidar. Boundary-Layer Meteorol 127:111-130

Raupach M, Antonia R, Rajagopalan S (1991) Rough-wall turbulent boundary layers. Appl Mech Rev 44:1-25

Rayleigh L (1916) On convection currents in a horizontal layer of fluid, when the higher temperature is on the under side. Philos Mag Ser 32(192):529-546

Robinson S (1991) Coherent motions in the turbulent boundary layer. Annu Rev Fluid Mech 23:601-639

Ruck B (1987) Laser Doppler anemometry—a non-intrusive optical measuring technique for fluid velocity. Part Part Syst Charact 4:26-37

Salesky ST, Katul GG, Chamecki M (2013) Buoyancy effects on the integral length scales and mean velocity profile in atmospheric surface layer flows. Phys Fluids 25(10):105,101

Segalini A, Alfredsson P (2012) Techniques for the eduction of coherent structures from flow measurements in the atmospheric boundary layer. Boundary-Layer Meteorol 143:433-450

Stawiarski C, Träumner K, Knigge C, Calhoun R (2013) Scopes and challenges of dual-Doppler lidar wind measurements_-an error analysis. J Atmos Ocean Technol 30(9):2044-2061

Stull R (1988) An introduction to boundary layer meteorology. Kluwer, Dordrecht 666 pp

Takimoto H, Inagaki A, Kanda M, Sato A, Michioka T (2013) Length-scale similarity of turbulent organized structures over surfaces with different roughness types. Boundary-Layer Meteorol 147:217-236

Young GS, Kristovich D, Hjelmfelt M, Foster R (2002) Rolls, streets, waves and more: a review of quasi-twodimensional structures in the atmospheric boundary layer. Bull Am Meteor Soc 83:997-1001

Zeeman MJ, Eugster W, Thomas CK (2013) Concurrency of coherent structures and conditionally sampled daytime sub-canopy respiration. Boundary-Layer Meteorol 146:1-15

Zhang Y, Heping L, Foken T, Williams Q, Mauder M, Thomas CK (2010) Coherent structures and flux contribution over an inhomogeneously irrigated cotton field. Theor Appl Climatol 103(1-2):119-131 